Archives de sciences sociales des religions

147 | juillet-septembre 2009

Traduire l'intraduisible

\title{
Roman Jakobson et la traduction des textes bibliques
}

Michaël Oustinoff

\section{(2) OpenEdition}

1 Journals

Édition électronique

URL : http://journals.openedition.org/assr/21368

DOI : $10.4000 /$ assr. 21368

ISSN : $1777-5825$

Éditeur

Éditions de l'EHESS

Édition imprimée

Date de publication : 1 octobre 2009

Pagination : 61-80

ISBN : 978-2-7132-2217-7

ISSN : 0335-5985

Référence électronique

Michaël Oustinoff, «Roman Jakobson et la traduction des textes bibliques », Archives de sciences sociales des religions [En ligne], 147 | juillet-septembre 2009, mis en ligne le 01 octobre 2012, consulté le 19 avril 2019. URL : http://journals.openedition.org/assr/21368 ; DOI : 10.4000/assr.21368

() Archives de sciences sociales des religions 


\section{Michaël Oustinoff}

\section{Roman Jakobson et la traduction des textes bibliques}

Qui n'a pas entendu parler du célèbre article de Roman Jakobson intitulé «Aspects linguistiques de la traduction » (Jakobson, 1963) ? Rares en tout cas sont les études sur la traduction qui ne le citent pas : il fait référence en la matière et figure, à ce titre, en bonne place dans la Routledge Encyclopedia of Translation Studies (Baker, 2001). On y cherchera cependant en vain la trace de bien d'autres écrits du même auteur sur la question, rassemblés dans le sixième volume (Jakobson, 1985) de ses Selected Writings: Early Slavic Paths and Crossroads - soit près de quatre cents pages - consacré, pour l'essentiel, à la traduction des textes bibliques par Cyrille et Méthode au IX siècle qu'il ne craint pas de mettre en parallèle aussi bien avec la Septante, la Vulgate ou la Bible de Luther. Il y a donc là une faille à combler ${ }^{1}$, ne serait-ce qu'en rappelant l'existence et le contenu de ces travaux, encore peu connus au-delà du cercle des slavisants.

Une telle entreprise a un triple intérêt. Tout d'abord, elle permet de se faire une idée plus juste des conceptions jakobsoniennes en matière de traduction et de leur étendue : loin d'être une dimension mineure de son œuvre, elles en constituent au contraire une facette majeure. On ne s'étendra pas sur ce point qui demanderait à lui seul une étude entière.

Il y a une deuxième raison qui confère à ces écrits une importance toute particulière : si une traduction est analysable en fonction de ses « aspects linguistiques ", c'est une approche bien réductrice que de s'en tenir là et singulièrement dans le cas de la traduction des textes religieux, car l'auteur souligne que la tradition cyrillo-méthodienne exige d'être tout autant appréhendée sous l'angle de ses implications historiques, politiques et culturelles que proprement liturgiques. C'est ce qui fait la modernité de ces écrits (les premiers remontent aux années vingt) du point de vue de la traductologie des textes bibliques et des analyses les plus récentes (Lassave, 2005 ; Léonas, 2007).

1. Je remercie vivement Konstantin Sigov, Université de Kiev-Mohyla Akademie, de m'en avoir appris l'existence.

ARCHIVES DE SCIENCES SOCIALES DES RELIGIONS 147 (juillet-septembre 2009), pp. 61-80 
Mais il y a un troisième point, plus novateur encore, dans la mesure où la vision de la langue sur laquelle s'appuie Jakobson n'est pas celle qu'une longue tradition gréco-romaine nous a léguée, où la langue n'est que l'instrument passif de la pensée, mais celle développée notamment par un Wilhelm von Humboldt qui fait de la langue une force agissante et créatrice, une energeia, rejoignant ainsi des études récentes (Cassin, 2004 ; Nowicki, Oustinoff, 2007). Ce serait une erreur de ne considérer Early Slavic Paths and Crossroads que du point de vue de l'histoire des idées : il constitue un outil essentiel pour la recherche, notamment en ce qui concerne l'analyse de l'intraduisible (Derrida, 1985). Telles sont les différentes facettes que l'on examinera tour à tour.

\section{La tradition cyrillo-méthodienne dans la traduction occidentale}

La traduction de la Bible occupe une place centrale dans la genèse de la civilisation occidentale ${ }^{2}$, caractérisée par ses racines judéo-chrétiennes. Eugène Nida, suivant en cela une habitude communément admise, découpe l'histoire des traductions des textes bibliques en trois périodes (Baker, 2001: 23-24) : la période gréco-romaine, dont les principaux temps forts sont la traduction de la Bible des Septante par la communauté juive d'Alexandrie à partir du III siècle av. J-C. (en grec) et la Vulgate de saint Jérôme au IV siècle ap. J.-C. (en latin); vient ensuite la période de la Réforme, caractérisée par la traduction de la Bible dans les langues vernaculaires, dont les exemples les plus marquants sont la Bible de Luther (en allemand) et l'Authorized Version (en anglais); enfin, la troisième et dernière période est la période dite "moderne ", à partir essentiellement du $\mathrm{XIX}^{\mathrm{e}}$ siècle, où soit les traductions antérieures tendent à être révisées (première phase), soit de nouvelles traductions sont effectuées dans des langues dans lesquelles la Bible n'avait pas encore été traduite (seconde phase : en particulier les langues du «Tiers-Monde »).

En réalité, ces trois périodes s'ordonnent à partir d'un seul axe, celui du passage des trois langues véhiculaires originelles (hébreu, grec, latin) aux langues vernaculaires. Il y aurait ainsi un seul et même mouvement : la Septante aussi bien que la Vulgate avaient la même fonction, celle d'assurer la plus grande diffusion possible aux textes sacrés. En ce qui concerne la Septante, aussi bien pour le monde hellénistique qu'ensuite pour l'Empire romain d'Orient au regard du grec ; et réciproquement, en ce qui concerne la Vulgate, pour l'Empire romain d'Occident au regard du latin. C'est la situation qui a prévalu jusqu'à la Réforme, époque à laquelle la traduction dans les langues vernaculaires (et pas seulement des textes religieux : on peut faire remonter à la Renaissance ce grand mouvement de traduction dans le monde occidental) préfigure l'émergence des Étatsnations. Dans le cas de la traduction de la Bible par Luther, l'entreprise n'a

2. Terme entendu ici au sens élargi, par opposition à des aires culturelles comme celles de la Chine ou de l'Inde. 
pas seulement une signification linguistique, celle du simple passage du latin à l'allemand et des problèmes de traduction qui l'accompagnent, mais également des implications historiques, politiques et culturelles qu'il n'est pas besoin de développer ici tant elles sont connues, et l'on pourrait en dire autant de l'Authorized Version pour le monde anglo-saxon.

C'est contre une telle vision des choses, encore largement présente aujourd'hui au sein des Translation Studies (Oustinoff, 2007), que s'insurge Jakobson dans «The Beginning of National Self-Determination in Europe » $1985: 115-128)$, car elle trahit une totale méconnaissance des spécificités de ce que l'on appelle l'Autre Europe, méconnaissance qui est loin d'avoir disparu encore aujourd'hui (Nowicki, 2008) :

This historical schema is usually considered as the common European pattern for the birth and growth of the national idea. But, in reality, such a Pan-European evolutionary scheme is a pure fiction, a hasty generalization which is in accord with the facts only in the history of those European peoples who belong entirely to the occidental political and cultural world. This one-sided schema has to be substantially revised in dealing with the history of the peoples who were at least temporarily influenced by the Greek Empire, by the Byzantine cultural radiation. (Jakobson, 1985 : 115).

C’est en plaquant un tel schéma sur la partie médiane et orientale de l'Europe, en réalité calqué sur ce qui n'est vérifié que dans sa partie occidentale, que l'on passe à côté d'un fait souvent méconnu : loin de constituer un événement nouveau, coïncidant avec la Réforme, la traduction de la Bible par Luther ou l'Authorized Version ont été précédées de près de cinq siècles par les traductions de Cyrille et de Méthode dont les "fondements idéologiques » (ideological fondations) sont, à bien des égards, comparables, puisqu'ils reposent sur le principe suivant : " the equality of all languages and peoples and the sacred right of any vernacular tongue to be used for all spiritual tasks up to the Holy Communion » (Jakobson, 1985 : 108). Au IX siècle, à une époque où le politique et le religieux sont intimement imbriqués (qui dit égalité des langues dit égalité des peuples, donc des nations), ce principe est loin d'aller de soi. Pour Rome, seules trois langues peuvent être considérées comme sacrées, car elles auraient figuré toutes trois sur la croix du Christ: tres sunt autem linguae sacrae, his enim tribus linguis super crucem Domini a Pilato fuit causa eius scripta (Jakobson, ibid.) Pour Byzance, plus tolérante en la matière ${ }^{3}$, l'entreprise était moins ardue, mais l'argument décisif aura été (comme pour Rome, qui aura accordé son assentiment : Cyrille meurt, ne l'oublions pas, dans cette ville) la nécessité d'évangéliser des peuples vivant aux marges de l'Empire.

3. Cette plus grande tolérance s'explique pour des raisons historiques et géopolitiques: "Constantinople, la "Nouvelle Rome" créée par Constantin sur le site de l'antique Byzance, ne parvint jamais à assurer de manière aussi contraignante [que Rome] son droit d'intervention auprès de communautés dont les origines pouvaient souvent s'enraciner jusqu'aux premiers temps chrétiens et qui vivaient en des pays de très anciennes cultures, telle l'Égypte ou la SyrieMésopotamie, fiers de leur tradition, de leur langue, et attachés à leurs particularismes » (Poupard, $1984: 450)$. 
Mais Cyrille est le nom ecclésiastique de Constantin le philosophe : il n'est donc pas en manque d'arguments théologiques et se réclame de la première Épître aux Corinthiens de saint Paul et du Miracle de la Pentecôte où les Apôtres se voient accorder le don des langues. Prenant fait et cause contre les « trilingues », Cyrille leur oppose Mc 16,17 où il est dit que l'on utilisera contre les démons de «nouvelles langues»(Jakobson, 1985 : 110).

Mais la "tradition cyrillo-méthodienne» va plus loin, en renversant la perspective : au début du $\mathrm{X}^{\mathrm{e}}$ siècle, par exemple, le moine Xrabr proclame la supériorité de l'alphabet slave élaboré par Cyrille (l'alphabet glagolitique ${ }^{4}$ ) sur l'alphabet grec, car le premier est une création chrétienne conçue à des fins pieuses alors que le second est né dans un monde païen. Pour des raisons sans doute de commodité et de prestige liées au rayonnement du grec dans l'Empire byzantin, l'alphabet dit cyrillique est pour sa plus grande partie calqué sur l'alphabet grec, mais il ne faut pas oublier que telle n'était pas l'intention première. En créant un alphabet nouveau, était affirmée la volonté de se démarquer, dans le choix même des signes utilisés, des trois langues tenues jusqu'alors pour sacrées. Un parallèle peut ici être établi avec la divergence de vues opposant saint Jérôme et saint Augustin sur la valeur à accorder à l'original hébreu de l'Ancien Testament par rapport à la traduction de la Septante, telle qu'on la trouve exposée dans l'article «traduire » du Vocabulaire européen des philosophies (Cassin, 2004 : 1312) :

Contemporain et correspondant de Jérôme, Augustin rejette la règle de l'hebraica veritas. Pour lui, le texte grec des Septante est «inspiré par l'Esprit saint » : c'est la meilleure version qui soit. Ce qui signifie que, si vérité originelle il y a, c'est en elle qu'elle se trouve. Cette Bible grecque a vraiment annoncé le Christ (par exemple, en introduisant l'adjectif parthenos (...), "vierge ", pour traduire le mot hébreu signifiant « jeune femme ", à propos de la mère de l'Emmanuel, en Isaïe, 7,14), et l'Église a fait sienne cette traduction. Augustin croyait au progrès de l'humanité dans l'histoire, que le Christ, dernière étape, ne fait qu' « achever ».

Autrement dit, l'essentiel n'est pas tant d'évaluer dans quelle mesure la traduction est fidèle ou non à l'original (c'est le point de vue de saint Jérôme qui d'abord révise les traductions latines existantes à la lumière de la Septante, pour ensuite partir du texte hébraïque, en revenant ainsi «aux sources » (ad fontes) de la vérité révélée, l'hebraica veritas, mais de s'intéresser à la traduction en tant que texte à part entière, ou, pour emprunter cette jolie formule à Northrop Frye : "Clearly, then, one of our first problems is to determine the positive reality of translation, the essential thing or force or process that translation translates " $(1984: 4)^{5}$. C'est une telle «force » que déploiera Luther, qui surmontera ses

4. L'alphabet créé par Cyrille n'est pas, contrairement à ce que l'on pourrait penser, l'alphabet cyrillique : celui-ci, postérieur à l'alphabet glagolitique, est en réalité l'œuvre de saint Clément.

5. "Il est donc clair qu'un des premiers problèmes que nous ayons à résoudre est de déterminer quelle est la réalité positive de la traduction, l'objet ou la force ou l'opération essentielle que traduit la traduction»(Frye, 1984 : 42 ; trad. Catherine Malamoud). 
réticences à traduire la Bible en se rangeant à l'avis des hussites tchèques, euxmêmes adeptes de la tradition cyrillo-méthodienne en la matière : il y a par conséquent une continuité (le plus souvent méconnue) entre la traduction de Cyrille et de Méthode et celle de Luther ou l'Authorized Version (Jakobson, 1985 : 127). Mais cette force, qui est d'ordre idéologique, historique et culturel, est liée à la fois aux ressources de la langue dans laquelle s'effectue la traduction et de celle de l'original : c'est ce versant qui constitue l'axe majeur des analyses de Jakobson.

\section{Matrix omnium linguarum}

Pour saint Jérôme, la langue hébraïque était la matrice de toutes les langues (matrix omnium linguarum) : "la première dont toute autre dérive, au risque, à chaque moment, d'une déperdition de la vérité »(Cassin, 2004 : 1311). Rejoignant en cela saint Augustin, il se considère investi d'un savoir supérieur à celui dont disposaient les traducteurs de la Septante, dans la mesure où il est né après la venue du Christ. Mais s'il estime qu'il faut remonter aux sources, à l'hebraica veritas, c'est qu'il appuie sa philosophie du langage sur les écrits d'Origène et sur le Cratyle de Platon : l' "être " (grec on) et le "nom» (grec onoma) sont pour lui indissociables, et "cette union est réalisée avec le maximum de force dans la langue hébraïque, idiome primordial apte plus que tout autre à exprimer et garantir la vérité » (ibid.) Voilà pourquoi ce n'est pas la Septante qu'il s'agit de prendre pour référence, afin que les livres qu'il traduit ne soient pas « corrompus par le transfert dans un troisième vase », car " $[\mathrm{r}]$ emis à une cruche très proche dès la sortie du pressoir, ils y conservent leur saveur » (ibid.) Pour Augustin, la distinction entre on et onoma est secondaire par rapport à celle établie par la doctrine stoïcienne entre res et signa : la vérité de Dieu est toute entière contenue dans la res, et non dans les signes de la langue (Cassin, 2004 : 1312). Ce n'est pas sur ce terrain que s'aventure Jakobson, car il reste sur celui de la «matrice de la langue », au sens que lui donne Edward Sapir, qu'il cite (1985: 64) : "The literature fashioned out of the form and substance of a language has the color and texture of its matrix. » On reconnaît là l'hypothèse dite de SapirWhorf, qui établit que chaque langue constitue une "vision du monde » qui lui est propre, thèse déjà défendue par Wilhelm von Humboldt au XIX siècle, comme dans la citation suivante, rédigée directement en français par lui-même (Trabant, 1999 : 82) et qui n'est pas sans rappeler la citation précédente: "Toutes les langues ensemble ressemblent à un Prisme, dont chaque face montrerait l'univers sous une couleur diversement nuancée ». Il n'est pas exagéré de dire que l'ensemble de Early Slavic Paths and Crossroads doit se lire à la lumière d'une telle conception, matricielle, de la langue. Or il se trouve que, pour l'ensemble des langues slaves, la traduction de Cyrille et Méthode représente ce que représente la traduction de la Bible par Luther pour l'allemand, celle de l'Authorized Version pour l'anglais : une matrice essentielle, pour ne pas dire la matrice des matrices. C'est 
le cas, tout d'abord, au sens le plus étroit que l'on puisse donner au mot « langue ». En traduisant la Bible, Cyrille et Méthode fondent non seulement la langue liturgique, mais également la langue littéraire et philosophique du monde slave tout entier car, au IX ${ }^{\mathrm{e}}$ siècle, les langues slaves s'étaient très peu différenciées les unes des autres, contrairement aux langues romanes à la même époque.

La profonde unité du monde slave dérive de là, et encore aujourd'hui, selon Jakobson : "It is his mother tongue which reliably indicates that a man belongs to the Slavic world $(1985: 3)^{6}$. Néanmoins, sa langue est marquée (sans qu'il en ait conscience) par la matrice d'une autre, car cette langue a été forgée par la traduction :

Old Church Slavonic shaped its vocabulary, phraseology, style, and even some grammatical devices after the model of Greek. In standard Russian even now the effects of this patterning have remained so significant that a scholar and polyglot of the stature of Henri Grégoire can declare that it is sometimes easier to translate a passage of Leskov or Dostoievskij into Greek than into French or English. The Christian offshoot of Greek classical culture penetrated deeply into the Slavic world by means of Church Slavonic. (1985: 50).

L'empreinte laissée par le grec est si forte qu'elle n'a pas épargné l'autre branche de la culture classique de la chrétienté, la tradition latine, quand il s'est agi de la traduire : " un grand nombre des termes latins ont été hellénisés, les noms propres latins ont été régulièrement remodelés selon les normes grecques, certains procédés stylistiques byzantins ont été introduits en contrebande, et parfois même ce sont des extraits en grec que l'on a insérés » (Jakobson, 1985 : $50)^{7}$. De ce point de vue, on peut établir un parallèle entre ces analyses et celles contenues dans La Bible grecque des Septante. Du judaïsme hellénistique au christianisme ancien (Dorival et al., 1999), parallèle d'autant plus justifié que c'est à partir de la Septante que Cyrille et Méthode (et ensuite leurs continuateurs) ont traduit l'Ancien Testament (ibid.) L'ouvrage consacre un chapitre entier à la langue de la Septante, dont le sixième chapitre a un titre on ne peut plus parlant : "Y a-t-il eu "hellénisation" de la Bible hébraïque dans la langue grecque ?» (ibid.: 254). Les choix opérés par les traducteurs de la Septante ne sont pas sans incidences. C'est le cas, par exemple, pour la traduction par «Dieu » et par «Seigneur»:

Avec le nom théos, remplaçant «Elohim » et le mot kúrios employé pour YHWH, le Dieu personnel du peuple hébreu devient sans aucun doute la divinité universelle : les origines polythéistes ou du moins ethniques de ces noms disparaissent au profit d'un monothéisme accentué. (ibid. : 255).

6. "C'est sa langue maternelle qui démontre véritablement qu'un homme appartient au monde slave» (notre traduction).

7. « Many of the Latin terms were Hellenized, Latin proper names were regularly remodeled in the Greek manner, certain Byzantine stylistic devices were smuggled in, and at times even Greek excerpts were inserted » (notre traduction). 
À l'inverse, le grec des traducteurs contient des tours manifestement calqués sur l'hébreu, au risque de créer des opacités :

Ayant adopté le mot grec eiréne comme équivalent de l'hébreu shalom, "paix ", le traducteur emploie ce mot dans des tours qui décalquent les formules hébraïques comportant le mot shalom, pour signifier, par exemple, "demander des nouvelles". Le lecteur hellénophone a la surprise de lire, par exemple, en $2 \mathrm{R} 11,7$, que David interroge Uri (littéralement) "pour la paix de Joab, pour la paix du peuple et pour la paix de la guerre »(!), ce qui signifie, dans l'esprit du traducteur bilingue, qu'il " demande des nouvelles de Joab, des nouvelles du peuple, des nouvelles de la guerre ». (ibid: 249).

La Septante comporterait ainsi deux défauts concomitants : elle hellénise le texte original hébreu tout en hébraïsant le grec de la traduction, tant et si bien qu'elle ne saurait constituer un «texte autonome » : "Un large discrédit a longtemps affecté la LXX [Septante] comme œuvre littéraire : elle n’est qu'une traduction, utilisant trop de tours étrangers à la langue grecque (les littéralismes) pour qu'on lui reconnaisse une valeur littéraire "(ibid. : 259). Ce discrédit remonte en réalité au VII ${ }^{\mathrm{e}}$ siècle et n'est pas universel : "A partir du VII siècle, la conception de Jérôme, qui prône le retour à l'hebraica veritas, l'emporte dans le monde latin, mais sans pouvoir totalement éliminer la LXX : les Églises latines conservent leurs antiques Psautiers traduits sur le grec. De plus, cet effacement est contrebalancé, en Occident, par l'apparition, au $\mathrm{IX}^{\mathrm{e}}$ siècle, de la traduction vieux slave faite sur le grec et, en Orient, par l'importance grandissante de la LXX » (ibid. : 333-334). On rappellera, par exemple, que la Septante demeure la Bible des Églises orthodoxes, que ce soit en grec ou sous la forme d'une traduction (ibid. : 334). Par ailleurs, tout dépend du point de vue adopté. C'est ainsi que Jean Coste, dès 1954, partant du texte grec d'Isaïe 25,1-5, relève dans les cinq premiers versets une série impressionnante de " contresens » par rapport à l'original hébreu, mais il démontre que la traduction n'est pas « le résultat fortuit d'une juxtaposition de contresens de détail ; un souffle le traverse de part en part qui en fait une unité littéraire incontestable " (ibid. : 260) si bien qu'entre les différentes parties du poème apparaît "un réseau serré de reprises et de rappels » et qu'un texte qui semblait de prime abord un échec patent "se révèle, une fois reconsidéré pour lui-même, une composition ordonnée et cohérente ". Voilà pourquoi les auteurs de l'ouvrage parviennent à la conclusion suivante :

Mais si l'on est persuadé que toute traduction est à son tour un " texte ", pourquoi ne pas observer les « effets » de ce texte ? Ce que les traducteurs de la Bible hébraïque réclament avec raison (comme le fait brillamment $\mathrm{H}$. Meschonnic), à savoir que la totalité du message soit rendue dans les langues modernes, en préservant la forme qu'il revêt, ne doit-on pas l'accorder à la LXX, cette Bible qui fut vivante et productive dans le judaïsme hellénistique, puis dans les Églises anciennes et jusque dans les Églises modernes de rite grec? (Dorival et al., 1994 : 266).

L'évaluation de la Septante, en tant que "texte autonome ", est rendue délicate du fait qu'elle a été, dès l'Antiquité, l'objet de nombreuses controverses, 
aussi bien au sein du judaïsme (d'abord admirée, ensuite rejetée) que du christianisme (retour à l'hebraica veritas prônée par Jérôme). Il en va tout autrement pour la traduction de la Bible cyrillo-méthodienne dans le monde slave : elle s'est imposée comme un monument à la fois liturgique et littéraire. C'est pourquoi Jakobson peut pleinement analyser cette traduction sous l'angle de la poétique, une poétique dont la matrice se déploie sur deux langues à la fois.

\section{Poétique de la langue et traduction des textes bibliques}

Dire qu'une traduction de la Bible (ou plus généralement de tout texte sacré) puisse être investie d'une qualité littéraire, voire être considérée comme une œuvre littéraire n'a rien de spectaculaire : on le sait depuis longtemps dans le cas de la Vulgate (Larbaud, 1944), de la Bible de Luther (Berman, 1984), ou de l'Authorized Version (Frye, 1983 ; Alter et al., 1987), etc. En revanche, plus rares sont les études qui analysent dans le détail la genèse d'une telle littérarité (Meschonnic, 1999 ; Lassave, 2005) : c'est là une originalité majeure de Early Slavic Paths and Crossroads. Éparpillée sur plusieurs articles à la fois (et en plusieurs langues : anglais, français, russe, polonais, tchèque), la démarche de Jakobson pourrait se résumer à trois principes : 1- chaque langue porte en elle une poétique qui lui est propre ; 2- la traduction slave de la Bible est une œuvre poétique ; 3 - la poétique de cette traduction est indissociable de celle de l'original en langue grecque.

Le premier point n'a pas besoin d'être développé ici car les thèses de Jakobson sur la question sont suffisamment connues : elles remplissent, à elles seules, le troisième volume des Selected Writings, intitulé Poetry of Grammar and Grammar of Poetry (1981). Le second point pourrait également être mentionné au passage, tellement il semble aller de soi dans le monde slave : sa valeur littéraire n'a rien à envier à celle de la Bible de Luther ou de l'Authorized Version. Qui plus est, dans l'article "St Constantine's Prologue to the Gospels » (1985 : 191206), Jakobson insiste sur le fait que Constantin, en traduisant, fait œuvre de poète, car il est, lui-même, poète :

From the initial to the final lines of the Proglas the poet remains faithful to the masterful architectonics of sound and sound [sic] sequence, morpheme and word, repetition and variation. Constantine's power in the literary language he created is indeed amazing; it embraces the whole diversity of functions assigned to the new tool [...]. The philosopher deploys the brightest poetic figures of the Byzantine world in order to affirm and deepen the sovereignty and equality that are bestowed on every nation and on everyone within each nation as soon as the native word has found its access to the Holy Communion, which, in the Middle Ages, was conceived as the acme of attainable communication. (ibid. : 205).

Son Prologue (russe Proglas) à la traduction des Évangiles est soumis à une explication de texte en bonne et due forme et, pour que l'analyse soit accessible 
à tous les lecteurs anglophones, le texte original est accompagné d'une traduction en anglais par l'auteur. Sans vouloir reprendre in extenso l'analyse (résumée d'ailleurs dans la citation précédente), on retiendra un élément-clé qui fait l'objet de la première partie de l'article, à savoir la métrique : le mètre utilisé est calqué sur le dodécasyllabe byzantin (1985 : 193). Sa structure se caractérise par : 1- un «pont » (zeugma) servant de repère et situé exactement au milieu du vers, après la sixième syllabe ; 2- l'ajout d'une césure à une syllabe de distance du pont, c'est-à-dire après la cinquième (césure marquée par /) ou la septième syllabe (marquée par //) : on peut avoir aussi bien des vers " ascendant » de type $5+7$, les plus fréquents, des vers au contraire "descendants ", de type $5+7$, mais on peut trouver des vers à deux césures. Pour les vers 19 et 20, cela donne par exemple (ibid. : 199) :

\section{Grěxovъnojo / tъmo // otъgъnati}

20. I mira sego / ţlo // oţložiti
To dispel the darkness of sin And to repel the corruptness of this world ${ }^{8}$

La structure de ces deux vers est la même, $5+2+5$, où au centre on trouve deux accusatifs (tъmo "ténèbres " et $t$ Ђlo «corruption»), symétrie renforcée, sur le plan des signifiants, par les allitérations et les assonances, et sur le plan des signifiés, par le parallèle établi entre «ténèbres » et «corruption »; il en va de même pour les deux infinitifs placés en finale otъgъnati ( dispel ») et otъložiti ( « repel »). L'article suivant, intitulé « The Slavic Response to Byzantine Poetry » (1985 : 240-259) constitue la clé initiale, en l'occurrence une découverte majeure faite par Jakobson dès 1917 :

In 1917, while examining the oldest Russian records of church songs, I was amazed by their clear-cut rhythm in striking contradiction to the then current philological allegation that the Slavic translations of the Greek hymns were in prose, and that poetry was altogether neglected in Church Slavonic literature. (ibid. : 241).

Ce n'est que bien plus tard, à partir des années cinquante, que s'opère finalement un véritable tournant (turning point), qui, par des publications de plus en plus nombreuses, vient confirmer le point de vue déjà exprimé en 1896 par Wilhelm Meier au sujet de la poésie liturgique byzantine : «by far the most splendid and, along with historical writings, the most important part of Byzantine literature » (Jakobson, 1985 : 242) ${ }^{9}$. Auparavant, souligne Jakobson, la poésie byzantine était considérée comme une simple manifestation d'adresse formelle (formal jugglery), piétiste, guindée, désenchantée et dépourvue d'individualité (pietistic, stilted, contentless, and deprived of individuality). En réalité, cet art forme un tout, que le monde slave va non pas décalquer servilement, mais transformer de manière créatrice :

8. «19. Pour disperser les ténèbres du péché 20. Et repousser la corruption de ce monde » (notre traduction). Une traduction mot à mot serait «19. (Pour) du péché / les ténèbres//disperser 20. Et de ce monde / la corruption // repousser ».

9. «De loin la plus splendide et, parallèlement aux écrits historiques, la part la plus importante de la littérature byzantine » (notre traduction). 
The Slavic variant of Byzantine ecclesiastical art holds in poetry and music a position similar to its role in painting: it masters and transforms the Greek models. The pressure of a different verbal material acts as a complementary incentive to further modifications. Neither in poetry nor in the fine arts, however, does the adherence to the Byzantine pattern prevent originality. (ibid.: 242).

Par exemple, en comparant un chant grec et sa traduction en slavon, Jakobson montre une structure en miroir où, au découpage des soixante-dix syllabes de l'original en douze unités mélodiques correspond, à une syllabe près (soixanteneuf au lieu de soixante-dix), une distribution identique (Jakobson, 1985 : 243) :

\begin{tabular}{|l|c|c|c|c|c|c|c|c|c|c|c|c|}
\hline & 1. & 2. & 3. & 4. & 5. & 6. & 7. & 8. & 9. & 10. & 11. & 12. \\
\hline slavon & 5 & 6 & 8 & 8 & 6 & 5 & 7 & 7 & 4 & 5 & 3 & 5 \\
\hline grec & 5 & 6 & 8 & 8 & 6 & 5 & 8 & 7 & 4 & 5 & 3 & 5 \\
\hline
\end{tabular}

C'est le même mécanisme qui explique certains déplacements syntaxiques,

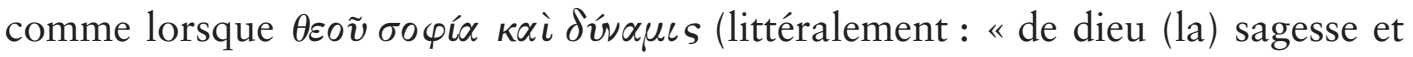
(la) force ») est traduit par božija sila i mudrostъ, où " sagesse » (mudrostъ) et "force» (sila) permutent. Cette inversion, au sein d'une structure comptant le même nombre de syllabes (neuf dans les deux cas), est en réalité due au souci de conserver le même schéma accentuel et mélodique : sila et $\sigma \circ \varphi i ́ \alpha$ sont accentués sur la pénultième syllabe, de même que mudrostъ et d'v́aucs le sont sur l'antépénultième.

Mais la recherche de telles correspondances - qui parfois aboutit à des effets plus heureux que dans l'original (ibid.: 245) - ne relève pas seulement de la littérature, mais des autres arts, que ce soit de la musique, dont il vient d'être question, ou de la peinture :

There are striking correspondences between Old Russian church poetry and painting: an impressive continuity, initiated and sustained, links this poetry with Byzantine artistic models, and simultaneously there appear early and continuous creative departures from former standards (whether foreign or native) and a variety of temporal and regional styles. (ibid. : 256).

La traduction des textes bibliques, du moins pour ce qui est du monde slave, s'inscrit donc dans ce que Peter Torop, en élargissant la perspective jakobsonienne, appelle la «traduction totale»(1995).

\section{Intraduisible et transposition poétique}

Ainsi qu'on l'a déjà signalé, les études portant sur la traduction des textes bibliques et s'appuyant sur la théorie de la traduction échafaudée par Jakobson ne manquent pas, mais elles ne citent presque jamais Early Slavic Paths and 
Crossroads et se focalisent sur le seul article «Aspects linguistiques de la traduction » (1963) ${ }^{10}$. Que cet article soit d'une importance capitale pour nombre de disciplines (études littéraires, linguistique, sémiotique, traductologie) ne fait aucun doute ; néanmoins, on risque, en le dissociant du plus grand ensemble que constitue l'ouvrage dans son ensemble, de fausser la perspective. Que retient-on, en effet, généralement de l'article ? Tout d'abord, la fameuse tripartition des formes de traduction possibles, axée sur la théorie peircienne des signes (Jakobson, $1963: 35)$ :

1) La traduction intralinguale ou reformulation (rewording) consiste en l'interprétation des signes linguistiques au moyen d'autres signes de la même langue.

2) La traduction interlinguale ou traduction proprement dite consiste en l'interprétation des signes linguistiques au moyen d'une autre langue.

3) La traduction intersémiotique ou transmutation consiste en l'interprétation des signes linguistiques au moyen de signes non linguistiques.

De ce point de vue, l'article mérite effectivement d'être inclus dans un recueil qui s'intitule Essais de linguistique générale : toute sa première partie est consacrée à ce qu'il est universellement possible de traduire, quelles que soient les langues considérées. La seconde partie, bien plus courte que la première, analyse l'autre pendant de la question qu'est l'intraduisibilité. Or, il y a fondamentalement deux manières de concevoir l'intraduisible : soit de manière négative, ce qui, à l'époque ou l'article a été rédigé, était la conception dominante ; soit, au contraire, de manière positive (Meschonnic, 1999 ; Berman, 1984 ; Cassin, 2004), point de vue de plus en plus largement partagé, mais bien minoritaire à l'époque. Décontextualisé, l'extrait suivant a été généralement interprété comme relevant de la première optique ; mais l'interprétation, semble-t-il, est tout autre si on le relie à la partie immergée de l'iceberg que représentent les écrits sur la traduction contenus dans Early Slavic Paths and Crossroads :

Il est très curieux que la toute première question qui fut soulevée dans la littérature slave à ses débuts fut précisément celle de la difficulté éprouvée par le traducteur à rendre le symbolisme des genres, et de l'absence de pertinence de cette difficulté du point de vue cognitif (...) « Le grec, traduit dans une autre langue, ne peut pas toujours être traduit identiquement, et c'est ce qui arrive à chaque langue quand on la traduit " dit l'apôtre slave [Constantin le philosophe]. «Des noms tels que potamos, "fleuve", et aster "étoile", masculins en grec, sont féminins dans une autre langue, comme reka et zvezda en slave ». D'après le commentaire de Vaillant, cette divergence efface l'identification symbolique des fleuves aux démons et des étoiles aux anges dans la traduction slave de deux versets de Mathieu (7:25 et 2:9). Mais à cet obstacle poétique, saint Constantin oppose résolument le précepte de Denys l'Aréopagite, selon lequel il faut d'abord être d'abord attentif aux valeurs cognitives (sile razumu), et non aux mots eux-mêmes. (1963: 85-86).

10. Il suffit, pour s'en apercevoir, d'entrer dans Google les mots-clés «traduction + bible + jakobson » (plus de trois mille résultats) et rajouter "Early Slavic Paths and Crossroads » : le nombre descend à deux pour le français, et à six pour l'anglais, etc. 
D'où la conclusion (souvent citée) à laquelle Jakobson parvient en matière de traduction poétique : «La poésie, par définition, est intraduisible. Seule est possible la transposition créatrice : transposition à l'intérieur d'une langue - d'une forme poétique à une autre -, transposition d'une langue à l'autre, ou, finalement, transposition intersémiotique - d'un système de signes à un autre, par exemple de l'art du langage à la musique, à la danse, au cinéma ou à la peinture » (ibid. : 86). À la lecture de cet article seul, on est tenté de voir dans l'intraduisibilité l'envers négatif de la traduction : un processus qui d'ailleurs relèverait davantage de l'insaisissable, en tout cas quelque chose qu'il serait bien plus difficile à étudier que le traduisible, tout entier analysable par les instruments dont disposent les humanités ou les sciences humaines et que systématise Jakobson dans la majeure partie de son article. Mais c'est en réalité tout le contraire qui est vrai : l'intraduisible n'est nullement pour lui une donnée négative, car il démontre exactement le contraire tout au long de Early Slavic Paths and Crossroads : la traduction des textes bibliques initiée par Constantin et poursuivie par la tradition cyrillométhodienne est toute entière du côté de la positivité, de la «transposition créatrice ", non pas en tant que concept général, mais en acte, et analysable en tant que tel.

Voilà qui explique que, loin d'être une version affadie de l'original, une traduction peut au contraire lui donner un second souffle, y compris pour l'auteur lui-même, comme lorsque Boris Pasternak se mettait à lire en tchèque la traduction de ses œuvres aussi bien en vers qu'en prose : "Through it his own past assumed a surprisingly new, yet kindred, shape, and he found in this transfigured past a new impetus to resume his creative path » (Jakobson, 1985 : 56). Par conséquent, considérer que la langue est un simple instrument de la pensée - suivant en cela l'opinion courante - n'est ici d'aucun secours : si tel était le cas, on comprendrait mal l'intérêt pour Pasternak de relire ses œuvres en traduction. À l'inverse, si l'on adopte le point de vue de Humboldt sur la question, tout s'éclaire. Dans un de ses écrits, celui-ci affirme au sujet de la langue, reprenant une distinction aristotélicienne: "Sie selbst ist kein Werk (Ergon), sondern eine Thätigkeit (Energeia)» (Humboldt, 2003 : 315), ce que l'on traduira par: "Elle-même n'est pas une œuvre (ergon) mais une activité (energeia) ». Autrement dit, la langue n'est pas un agent passif mais au contraire une force agissante (Cassin, 2004) : tous les écrits de Jakobson que l'on vient de passer en revue peuvent en être considérés comme l'illustration en acte.

De même qu'en physique un champ s'ordonne en fonction des forces élémentaires qui le caractérisent, le champ de la traduction gagne à être rapporté à ce concept de l'energeia humboldtienne. Cette force (Frye, 1984 : 4), toujours présente, se manifeste avec le plus d'intensité dans le cas d'une traduction considérée comme étant elle-même une œuvre littéraire, à plus forte raison poétique, comme l'est la traduction de la Bible par Cyrille et Méthode. De ce point de vue, l'un des plus grands apports de Jakobson est d'en avoir étudié les manifestations à 
ses différentes échelles, à commencer par celle de la langue et en fournissant une grille d'analyse fiable et généralisable à l'ensemble du domaine (ce que l'on ne trouve pas, à notre connaissance, dans les études portant sur l'hypothèse SapirWhorf (Sapir, 1921; Whorf, 1956 ; Benveniste, 1974), du moins de manière aussi systématisée.

On ajoutera que cette energeia cyrillo-méthodienne dépasse le cadre purement religieux, et trouve ses prolongements dans le monde moderne, y compris chez les auteurs athées : "The phraseology and imagery of the Church Slavonic literary tradition, is a vital stimulus for the Russian literary art of the twentieth century, to mention only Blok, Belyj, Kljuev, Esenin, and Majakovskij, however far from piety the last may appear to be "(Jakobson, 1985 : 47). Cette force agissante ne saurait par conséquent être cantonnée dans les limites traditionnelles où l'on a trop souvent tendance à enfermer le terme de "langue " (Oustinoff, 2008) ou celui de «traduction" (Nowicki, Oustinoff, 2007).

\section{Traduction et liturgie dans le monde orthodoxe}

Dans le cadre d'une vision aristotélicienne de la traduction où les langues sont interchangeables, que la Bible s'écrive en caractères hébreux, en caractères latins ou en caractères grecs, voire cyrilliques, est, tout compte fait, un épiphénomène. Si l'on se place au contraire dans le cadre d'une vision élargie de la traduction (Cassin, 2004), il en va tout autrement. Ainsi que l'a tragiquement mis en lumière l'éclatement de l'ex-Yougoslavie, les clivages entre populations de tradition catholique et de tradition orthodoxe s'inscrivent dans l'alphabet même utilisé pour retranscrire la langue : ce qui distingue le serbe du croate, c'est essentiellement le fait que le premier s'écrit en caractères cyrilliques et le second en caractères latins. La lettre, en ce sens, n'est nullement insignifiante.

Le schisme entre monde catholique et monde orthodoxe ne date que de 1054 : comme le rappelle Dimitri Obolensky, Cyrille et Méthode vivent à une époque où ces deux mondes se trouvent en paix plutôt qu'en guerre. Rome, loin de frapper d'anathème l'œuvre entreprise, finit par la voir d'un œil bienveillant : «It is the Frankish clergy, with its urge for cultural domination and political control, that destroyed the Slavonic vernacular Christianity that was planted in Central Europe by the common action of Rome and Byzantium » (1992: 213). La question de la lettre demande par conséquent à être rapportée, à l'origine, à un contexte différent, que l'on pourrait illustrer par le passage de l'alphabet glagolitique à l'alphabet dit « cyrillique ».

L'alphabet glagolitique était délibérément nouveau, afin qu'il se distingue des trois langues jusqu'alors tenues pour sacrées : l'hébreu, le grec et le latin. La thèse de Cyrille était en effet que ces trois alphabets, datant d'avant la venue du Christ, portaient en eux ce défaut pour ainsi dire originel. On attribue généralement le remplacement de l'alphabet glagolitique par l'alphabet cyrillique à 
Clément d'Okhrid, l'élève de Cyrille, qui lui aurait donné ce nom en hommage à son maître. Quoi qu'il en soit, si l'alphabet glagolitique ne fait, a priori, penser à aucun autre, l'alphabet cyrillique évoque immédiatement l'alphabet grec, dont il est directement issu. Au-delà de l'aspect purement linguistique, la lettre a ici valeur iconique, tout comme le latin qui, selon la formule de Françoise Wacquet, paraphrasant une formule de Rémy de Gourmont, a représenté « l'empire du signe » (Wacquet, 1998) dans le monde occidental. Dans sa partie orientale, cet empire s'incarne tout autant dans la graphie, y compris naguère en Roumanie, seul pays de tradition orthodoxe à utiliser aujourd'hui l'alphabet latin :

Le slavon, la variété d'ancien slave utilisée comme langue liturgique par l'Église orthodoxe, a été introduit au $10^{\mathrm{e}} \mathrm{s}$. en Roumanie, et il y a joué le rôle d'une langue de culture jusqu'au milieu du $17^{\mathrm{e}} \mathrm{s}$. L'accès à la scripturalité du roumain même est passé par ce canal : des premiers textes en langue vernaculaire (16 $\mathrm{s}$.) jusqu'au $19^{\mathrm{e}} \mathrm{s}$., le roumain s'est toujours écrit en caractères cyrilliques. On peut dire sans exagération que le slavon a rempli pour le roumain le rôle que le latin a joué pour la constitution des langues romanes d'Europe occidentale, et cela notamment dans l'élaboration du vocabulaire abstrait. (Buchi, 2003 : 1629).

La «scripturalité », pour reprendre ce terme à Eva Buchi, n'est donc pas insignifiante. C'est un lieu commun que de dire que la ligne de partage entre le monde orthodoxe, d'un côté, et le monde catholique et protestant, de l'autre, passe par l'adoption des caractères cyrilliques ou des caractères latins. Dans le même ordre d'idées, on ajoutera sans doute que la similitude des caractères cyrilliques et des caractères grecs ne fait que trahir le fait que l'évangélisation du monde slave au IX ${ }^{\mathrm{e}}$ siècle par Cyrille et Méthode s'est effectuée sous le signe de l'empire byzantin. Ce n'est pas faux, notamment en ce qui concerne la liturgie, tant celle-ci a frappé, par sa magnificence, l'esprit des contemporains :

"The liturgy of the Eastern Church is one of the great original creations of Byzantine genius. On the Russians of the Middle Ages it produced an impression of overwhelming beauty: "We knew not whether we were in heaven or on earth", so did the Russian envoys of Vladimir describe to their sovereign the service in St. Sophia in Constantinople in the late tenth century. " (Obolensky, 1994 : 215).

On ne saurait sous-estimer cette influence :

Translated into Slavonic, this liturgy, with its eucharistic drama, the poetry of its hymns, and the dogmatic symbolism of its setting-the church with its mosaics and frescoes depicting the heavenly hierarchy dominated by the majestic figure of the Pantokrator-Christ the All-Ruler-entered the very core of Slav Christianity. Its role was thus described by the British Byzantinist, the late Norman Baynes, in a lecture delivered in 1945 : "Still today it is the common liturgy which is the bond between the separate branches of the Orthodox Church-the liturgy in the vernacular tongue which was the gift of Byzantium" [...]. (Obolensky, 1994 : 215-216).

Néanmoins Jakobson démontre que l'on ne saurait s'en tenir à cette seule dimension, pour importante qu'elle soit : si la tradition cyrillo-méthodienne s'inscrit manifestement dans la tradition byzantine, elle n'en constitue pas une transposition purement mécanique, obtenue par simple décalque. Les recherches les plus récentes lui donnent en cela raison (Mitenova, 2004) : tout d'abord, au 
regard de la langue, non seulement quant à ses implications religieuses mais également conceptuelles et, plus largement, philosophiques : en «calquant » sur

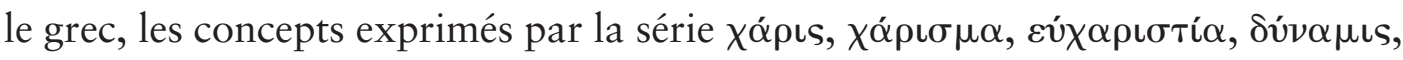

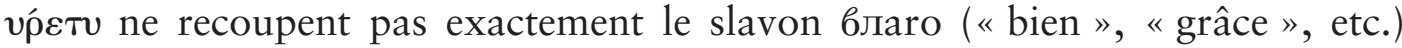
censé les rendre (Ovchinnikova, Chumakova, 2001 : 185) ; ensuite, et surtout, parce qu'à partir de cette idée sapirienne de la diversité matricielle des langues il est possible, en élargissant la perspective, d'englober tour à tour les autres points au sein d'une vision d'ensemble qui autrement ferait défaut. Au cours de leur mission évangélisatrice, Cyrille et Méthode n'ont pas fait que traduire des textes; c'est toute la liturgie du rite byzantin orthodoxe qu'ils ont introduite dans le monde slave, liturgie dans laquelle, ainsi que le souligne Jakobson, tout se tient : de l'hymnologie à l'architecture, en passant par la peinture. Il serait plus juste de parler de liturgie byzantino-slave, pour mettre en relief le fait qu'il ne s'agit pas d'une simple copie de l'original. Le film d'Andrei Tarkovsky, Andrei Rublev (1965), en est l'illustration frappante dans le domaine pictural : au lieu de s'inspirer servilement du style de Théophane le Grec, Andrei Rublev finit par trouver sa propre originalité tout en restant au sein de la même tradition.

Dans le cas de Cyrille et Méthode, vivant plusieurs siècles plus tôt, et en particulier avant le schisme de 1054, le mot de "tradition " risque, au singulier, d'être trompeur : le pluriel convient mieux. En effet, l'opposition entre Rome et Byzance n'était pas encore consommée :

" Be that as it may, there are cogent reasons for supposing that both the Roman and the Byzantine liturgies were translated into Slavonic in the second half of the ninth century. And it is quite possible that the liturgical tradition eventually adopted in the new Slavonic Church in Central Europe represented a blending of the Byzantine and the Roman rites »(Obolensky, 1994 : 208).

Non que les tensions entre la partie occidentale et la partie orientale de l'Empire romain n'aient pas été, à l'époque, perceptibles: le "mélange » des deux traditions est donc, par conséquent, délibéré et vise à les réconcilier. Enfin, il s'agit d'une synthèse créatrice et non de la simple juxtaposition de deux éléments :

If one were to attempt a general assessment of the work of Cyril and Methodius, its significance, I suggest, would be seen to lie in its unifying tendency and creative character. In a Christendom that was beginning to feel the growing tension between East and West, they sought to reconcile and to unite three important elements in the civilization of medieval Europe: the Byzantine, the Roman, and the Slavonic. (Obolensky, $2004: 212$ ).

On entre là dans un domaine qui, dira-t-on, se laisse difficilement saisir dans le cadre de l'energeia humboldtienne, puisque celle-ci s'applique avant tout à la langue, ou dans celui de l'article «Aspects linguistiques de la traduction » de Jakobson (op. cit.), à l'approche trop étroitement « linguistique ». Les Early Slavic Paths and Crossroads viennent opportunément rappeler la nécessité d'adopter une approche plus englobante et, pour tout dire, pluridisciplinaire, seule en mesure d'appréhender cette energeia-là. 


\section{Conclusion}

Les enseignements à tirer de Early Slavic Paths and Crossroads pour l'analyse de la traduction des textes sacrés sont si nombreux que le présent article ne saurait avoir la prétention d'en faire le tour. Cette limitation présente à l'esprit, on retiendra trois points principaux.

Tout d'abord, les analyses de Jakobson sur la traduction biblique dans le monde slave n'invalident nullement les ouvrages existants sur l'histoire de la traduction occidentale (Ballard, 2007), mais elles démontrent la nécessité d'intégrer l'étude de la tradition cyrillo-méthodienne sur un pied d'égalité avec celles portant sur la Septante, la Vulgate de saint Jérôme, la Bible de Luther ou l'Authorized Version. Il y a, dans cette conjonction, un champ de recherches encore aujourd'hui largement inexploré - du moins en dehors du cercle des slavisants.

Ensuite, ces analyses viennent confirmer les vues de ceux qui affirment l'impossibilité de traiter de la traduction littéraire - car la traduction de la Bible en vieux slavon d'Église est (aussi) un monument littéraire, qui constitue de surcroît le socle sur lequel s'est bâtie la littérature du monde slave dans son ensemble - en dehors de la poétique, ce qui implique qu'il y ait une poétique de la traduction (Meschonnic, 1999 ; Berman, 1995). En particulier, la Vulgate, la Septante, l'Authorized Version, la Bible de Luther sont, selon Henri Meschonnic, non seulement de "grand[s] chef-d'œuvre[s] » mais également des " originaux seconds ", ce qui est une "notion très complexe" (Houriez, 2001: 158) : on peut en dire tout autant des traductions de Cyrille et de Méthode, et l'analyse de Jakobson s'inscrit parfaitement dans cette (double) optique.

Enfin, et plus généralement, quelle que soit la valeur littéraire accordée à la traduction d'un texte religieux ${ }^{11}$, il est impossible de l'examiner sous un angle purement linguistique, poétique ou traductologique : en soulignant que les traductions de Cyrille et Méthode sont inséparables de leur portée historique, politique et culturelle (Obolensky, 1992 ; Flusin, 2006), Jakobson démontre la nécessité d'une approche véritablement pluridisciplinaire en la matière. Certes, un tel point de vue est devenu aujourd'hui monnaie courante parmi les spécialistes: mais n'est-ce pas là une nouvelle preuve de la modernité de l'héritage jakobsonnien et une raison de plus d'en redécouvrir le versant en langue russe, que l'autre, plus connu, en anglais ou en français, laisse injustement dans l'ombre?

11. Pour être tout à fait complet, il aurait fallu parler des autres traductions effectuées à partir de la Septante, comme la Bible de Wulfila en gothique (IVe siècle, autre monument littéraire), celles en arménien (début du V $\mathrm{V}^{\mathrm{e}}$ siècle), en géorgien ( $\mathrm{V}^{\mathrm{e}}$ siècle, d'abord à partir de la version arménienne, puis de la Septante), voire de la Peshitta en langue syriaque (celle-ci directement à partir de l'hébreu), mais cela nous aurait entraîné trop loin. 
Mais une telle "redécouverte" ne doit pas être passive : elle invite à une relecture novatrice à la lumière des recherches les plus récentes, car il n'y a pas de raison que les écrits de quelque auteur que ce soit, pas même Jakobson, restent paroles d'évangile. Ce serait le pire service à leur rendre.

\author{
Michaël OUSTINOFF \\ Université Paris III Sorbonne Nouvelle - Paris \\ michael.oustinoff@wanadoo.fr
}

\title{
Bibliographie
}

Alter Robert, Kermode Frank, (dirs.), 1987, The Literary Guide to the Bible, Cambridge, Harvard University Press.

Baker Mona, (dir.), 2001, Routledge Encyclopedia of Translation Studies, Londres, New York, Routledge.

Ballard Michel, 2007, De Cicéron à Benjamin. Traducteurs, traductions, réflexions, Lille, Presses Universitaires du Septentrion.

Benveniste Émile, 1974, "Deux modèles linguistiques de la cité ", in Problèmes de linguistique générale, 2, Paris, Gallimard, pp. 272-282.

BERMAN Antoine, 1984, L'épreuve de l'étranger. Culture et traduction dans l'Allemagne romantique, Paris, Gallimard.

-, 1995, Pour une critique des traductions : John Donne, Paris, Gallimard.

Buchi Eva, 2003, « Contacts linguistiques : langues slaves et langues romanes / Sprachkontakte : Slavisch und Romanisch" in Ernst G. et al. (dir.) Romanische Sprachgeschichte: ein internationales Handbuch zur Geschichte der romanischen Sprachen. Histoire linguistique de la Romania: manuel international d'histoire linguistique de la Romania, Berlin, New York, Walter de Gruyter, pp. 1627-1638.

CAssin Barbara, (dir.), 2004, Vocabulaire européen des philosophies. Dictionnaire des intraduisibles, Paris, Éditions du Seuil-Dictionnaires Le Robert.

Derrida Jacques, 1985, "Des tours de Babel ", in Graham J. K., (dir.), Difference in Translation, Ithaca, Cornell University Press.

Dorival Gilles, Harl Marguerite, MunNich Olivier, 1994, La Bible grecque des Septante. Du judaïsme hellénistique au christianisme ancien, Paris, Éditions du Cerf-CNRS Éditions.

Flusin Bernard, 2006, La civilisation byzantine, Paris, Presses Universitaires de France, coll. "Que sais-je ?", 3772.

FrYe Northrop, 1984, Le Grand Code. La Bible et la littérature, (préf. Tzvetan Todorov, trad. Catherine Malamoud), Paris, Éditions du Seuil [1983, The Great Code. The Bible and Literature, New York, Londres, Harvest, $1^{\text {re }}$ éd. 1981].

Houriez Jacques, (dir.), 2001, Claudel, le poète et la Bible, Besançon, Presses de l'Université de Franche-Comté.

Humboldt Wilhelm von, 2003, Über die Verschiedenheit des menschlichen Sprachbanes. Über die Sprache, Wiesbaden, Fourier Verlag. 
JaKOBSON Roman, 1963, "Aspects linguistiques de la traduction ", in Essais de linguistique générale, (trad. Nicolas Ruwet), Paris, Éditions de Minuit, pp. 71-86 ["On Linguistic Aspects of Translation ", in R. A. Brower, (dir.), 1959, On Translation, Cambridge, Harvard University Press, pp. 232-39].

-, 1981, Selected Writings: Poetry of Grammar and Grammar of Poetry. Vol. III, Berlin, New York, Mouton de Gruyter.

-, 1985, Selected Writings: Early Slavic Paths and Crossroads. Vol. VI, Berlin, New York, Mouton de Gruyter.

LARbaud Valery, 1944, [rééd. 1997], Sous l'invocation de saint Jérôme, Paris, Gallimard.

LASSAVE Pierre, 2005, Bible: la traduction des alliances. Enquête sur un événement littéraire, Paris, L'Harmattan, coll. "Logiques sociales".

LÉONAS Alexis, 2007, L'aube des traducteurs. De l'hébreu an grec : traducteurs et lecteurs de la Bible des Septante (III ${ }^{e}$ s. av. J.-C.-IV s. ap. J.-C.), Paris, Éditions du Cerf.

Meschonnic Henri, 1999, Poétique du traduire, Grasse, Verdier.

Mitenova Anisava, 2004, "The Cyrillo-Methodian Studies at the Beginning of the Twenty-First Century: Problems and Prospects », en ligne sur <www.slav.uni-sofia.bg/ liliJournal/archive/Lili1/MiltenovaA200401.html>

Nowicki Joanna, 2008, L'Homme des confins. Pour une anthropologie interculturelle, Paris, CNRS Éditions.

Nowicki Joanna, Oustinoff Michaël, 2007, Traduction et mondialisation, Hermès, 49, Paris, CNRS Éditions.

OвOLENSKy Dimitri, 1992, "Byzantium and the Slavic World ", in Laiou Angeliki E., Maguire Henry, (dirs.), Byzantium, a World Civilisation, Washington, Dumbarton Oaks, pp. 37-48.

-, 1994, Byzantium and the Slavs, Crestwood, St Vladimir's Seminary Press.

Ovchinnikova E. A., Chumakova T. V., 2001, "Ideas of "Blago" and "Dobro" in Russian Philosophy: From the Middle Ages to the Modern Times ", in Artemieva T., Mikeshin M., (dirs.), History of Ideas as a Methodology in the Humanities. The Philosophical Age. Almanac 18. Part 2, Saint Petersbourg, St. Petersburg Center for History of Ideas. En ligne (en russe) sur <http://ideashistory.org.ru/pdfs/25ovch_chum. pdf $>$.

Oustinoff Michaël, 2003 [2 éd. 2007], La traduction, Paris, Presses Universitaires de France, coll. "Que sais-je ?", 3688.

-, 2007, "Les "Translation Studies" et le tournant traductologique ", in Nowicki J., Oustinoff M., 2007, Traduction et mondialisation, Hermès, 49, Paris, CNRS Éditions, pp. 21-28.

-, 2008, "Traduction et energeia à l'heure de la mondialisation ", in Mitosek Z., CiesielskaRibard A., (dirs.), "Aspects sociologiques et anthropologiques de la traduction ", Les nouveaux cahiers franco-polonais, (Paris, Varsovie), 7, pp. 32-46.

Poupard Paul, (dir.), 1984, Dictionnaire des religions, Paris, Presses Universitaires de France

SAPIR Edward, 1921, Language. An Introduction to the Study of Speech, New York, Harcourt, Brace and Company. En ligne sur <http://www.bartleby.com/186/>

Trabant Jürgen, 1999, Traditions de Humboldt, (trad. Marianne Rocher-Jacquin), Paris, Éditions de la Maison des Sciences de l'Homme. [1990, Traditionen Humboldts, Frankfurt am Main, Suhrkamp] 
Torop Peeter, 1995, Total'nyj perevod [la traduction totale], Tartu, Tartu University Press.

WaCQUET Françoise, 1998, Le latin ou l'empire du signe, Paris, Albin Michel.

Whorf Benjamin Lee, 1956, Language, Thought and Reality, Cambridge, The MIT Press.

\section{Résumé}

Roman Jakobson a consacré de nombreux écrits à l'étude des textes bibliques traduits par Cyrille et Méthode, au IXe siècle, qui ont marqué d'une empreinte profonde l'ensemble du monde slave bien avant que des entreprises comparables voient le jour à la Renaissance dans le sillage de la Réforme (Bible de Luther, Authorized Version, etc.) Ces travaux, regroupés dans le sixième volume de ses Selected Writings, sont peu connus en dehors des slavisants, et ils sont d'une importance capitale non seulement pour les études slaves, la linguistique ou la traductologie mais également pour les sciences humaines en général. Euvre littéraire majeure, la traduction de la Bible par Cyrille et Méthode n'a pas qu'une dimension religieuse : elle unifie le monde slave autour d'une langue modelée sur le grec de la Septante et ne saurait être appréhendée qu'en fonction de ses autres implications historiques, politiques et culturelles.

Mots-clés : Traduction biblique, Septante, Cyrille, Méthode, Jakobson.

\section{Abstract}

Roman Jakobson devoted many of his writings to the translation of Biblical texts by Cyril and Methodius in the $9^{\text {th }}$ century, which had a huge impact throughout the Slavic world long before comparable attempts were made in the Renaissance in the wake of the Reformation (Luther's Bible, the Authorized Version, etc.). These papers, collected in the sixth volume of his Selected Writings, remain little-known except by Slavicists, yet they are of paramount importance not only for Slavic studies, linguistics and translation studies but also for other social sciences. Apart from being a major literary work in its own right, the translation of the Bible by Cyril and Method cannot be reduced to its religious dimension-it unified the Slavic world around a language fashioned after the Greek of the Septuagint and can only be fully understood in the light of its other historical, political and cultural implications.

Key words: Biblical translation, Septuagint, Cyril, Methodius, Jakobson.

\section{Resumen}

Roman Jakobson consagró muchos de sus escritos al estudio de los textos bíblicos traduzidos por Cirilo y Metodio en el siglo IX que marcaron profundamente todo el mundo eslávico mucho antes que surgiesen traducciones comparables en el Renacimiento durante la Reforma (la Biblia de Lutero, la Authorized Version, etc.). Estes trabajos, reunidos en el sexto volumen de sus Selected Writings, son poco conocidos excepto por los eslavistas pero tienen una importancia fundamental no solamente para los estudios eslávicos, la linguística y la traductologia sino también para las 
80 - ArChives de SCIENCES SOCIALES DES RELIGIONS

otras ciencias sociales. Obra literaria mayor, la traducción de la Biblia por Cirilo y Metodio no se puede reducir a la dimensión meramente religiosa: unificó el mundo eslávico en torno a una lengua modelada por el griego de la Septuaginta y es inseparable de sus otras implicaciones históricas, politicas y culturales.

Palabras clave: Traducción bíblica, Septuaginta, Cirilo, Metodio, Jakobson. 